\title{
Incidence of Clavicular Fractures and Its Management in Northern Ghana
}

\author{
Kuubiere B. Callistus ${ }^{1}$ and Alhassan Abass ${ }^{1 *}$ \\ ${ }^{1}$ Department of Human Biology, School of Medicine and Health Sciences, University for \\ Development Studies, Tamale, Ghana.
}

\section{Authors' contributions}

This work was carried out in collaboration between both authors. Author KBC designed the study, wrote the protocol, and analyses of the study. Author AA performed the statistical analysis, managed the literature searches and wrote the first draft of the manuscript. Both authors read and approved the final manuscript.

Research Article

Received $17^{\text {th }}$ February 2013

Accepted 23 ${ }^{\text {rd }}$ April 2013

Published $3^{\text {rd }}$ May 2013

\section{ABSTRACT}

Background: Fractures of the clavicle are common especially among young adults who are the work force in many developing countries, including Ghana. The incidence of clavicular fracture varies and the methods of treatment which is determine by the classification, age, fracture characteristics and the patients preference also varies although clavicular fractures are traditionally treated non-operatively. In the northern part of Ghana fractures are very common yet there has not been any study on the incidence of clavicular fractures and the preferred mode of treatment.

Aim: The aim of the present study is to retrospectively investigate the incidence of clavicular fracture and the preferred mode of management among fracture patients over a five year period.

Study Design: Retrospective

Place and Duration of Study: Tania specialist Orthopaedic Hospital, Tamale, Ghana. Methodology: Data regarding patient's age and sex, laterality involved, cause of injury and the mode of management of the fracture from January, 2007 to December, 2012 were analysed.

Results: The incidence of clavicular fracture over the study period was $12.8 \%$ of all fractured cases. Majority of the subjects $(71.4 \%)$ with clavicular fractures were aged 21 to 40 years. Old cases constituted $58.9 \%$ whiles only $41.1 \%$ of the cases were fresh fractures. About $70 \%$ of the old cases were people who had received management from 
traditional bonesetters. The fracture occurred on the clavicle of the right arm in $60.7 \%$ of the patients. Middle third fractures accounted for $78.6 \%$ with only $21.4 \%$ occurring on the distal third of the clavicle. The major cause of clavicular fractures in this study was road traffic accident constituting $83.0 \%$ followed by a fall from heights $11.6 \%$.

Conclusion: The incidence of clavicular fracture among the study subject was relatively high. Road traffic accidents were the most common cause of the injury and the preferred mode of management is the operative management using open reduction and internal fixation (ORIF).

Keywords: Clavicle; bonesetters; fracture; Northern Ghana.

\section{INTRODUCTION}

The clavicle which anatomically extends medially from the sternum to the acromion of the scapula is one of the most commonly injured bones in the body. It acts as a bridge connecting the upper limb to the trunk, transmitting forces placed on the upper limb to the axial skeleton and also functions as an attachment for various muscles [1]. Clavicular fractures are therefore associated with several impairments including loss of function of the upper limb [1]. Fractures of the middle third and lateral third of the clavicle account for approximately $80 \%$ and $15 \%$ of all clavicle fractures respectively, with only $5 \%$ occurring in the medial third [2-4].

The clavicle is generally fractured by two injury mechanisms. The most frequent type is a fall onto an outstretched arm or onto the lateral aspect of the shoulder where the force of the fall is transmitted through the upper extremity to the clavicle, producing the fracture. The other common mechanism of injury is direct trauma to the clavicle itself [5]. The type of clavicular fracture would thus depend on the mechanism of injury with most fractures of the lateral portion of the clavicle principally occurring after a fall onto the tip of the shoulder resulting in an injury similar to acromioclavicular joint dislocation [6]. Clavicular fracture is associated with pain and marked swelling at the site of fracture, decreased shoulder range of motion, brachial plexus nerve compression symptoms, and gross deformity of the clavicle [2].

In most settings the conventional immobilization with a sling or figure-of-eight strap is the method of choice for clavicular management regardless of the fracture configuration. However with potential complications such as frequent discomfort, breathing difficulties, and skin damage from tight straps from the figure-of-eight strap being high, most people now prefer to use the ORIF. Current management options available include open reduction and internal fixation of the clavicle plating with or without the use of bone grafting [4]. The mode of treatment of clavicular fracture is highly dependent on the site of fracture, age of patient, complexity of fractures and the degree of displacement. Recent evidence suggests that specific types of patients (athletes, patients with displaced fracture of more than $2 \mathrm{~cm}$ ) may be at high risk for nonunion, mal-union, shoulder dysfunction, or residual pain after nonsurgical management [7].

In Northern Ghana the first point of call for most fractures is at the Traditional bonesetter's home. It is only when they do not achieve the desired results that the individual would report to the orthopaedic centre. This leaves a lot of the patients with dysfunctional limbs due to nonunion or malunion at the fracture site. Because of the nature of clavicular fracture and the possibility of neuro-Vascular involvement due to displacement, it needs critical and adequate management to avoid persistent pain, possible neurological damage, and shoulder 
and upper limb impairments. Although it is generally known that clavicle fractures represent a common injury of the shoulder girdle in the general population, the true incidence in Northern Ghana is unknown. Given the implications of such injuries on the ability to perform occupation-specific tasks, determining the incidence in a population whose major occupation is farming is worthwhile. The main purpose of this study was to determine the incidence and management of clavicular fractures as well as the fracture pattern in Northern Ghana.

\section{METHODOLOGY}

The study was conducted retrospectively over a five year period at the Tania Specialist Hospital, Tamale from January 2007 to December 2012. All patients who presented to the hospital with clavicular fractures were included in the study. Patient characteristics (age, sex), fracture history (old case or new case), fracture characteristics (site of fracture, displacement and comminution) and cause of the injury (road traffic accident, fall from a height, or direct blow) were recorded. All old cases were further classified as having treatment obtained from a hospital/health centre or from a traditional bonesetter. A case was classified as old if the patient had received treatment either from the bonesetters or from any hospital prior to admission at the study site and fresh or new case if the patient had not received treatment or attention from any source and brought from accident site. All the new cases had fracture displacement of at least 2 centimetres as measured from the radiograph of the patient. These fractures were mostly complicated by wrong manipulations prior to arrival at the hospital. In this part of the country ambulance service is nonexistent and road traffic accident victim are usually carry to hospital in taxis or on motor cycle. The earliest reported old fracture was 18 weeks and the oldest fracture was 32 weeks. All the patients who reported as old cases were those associated with improper functioning of the upper limb, still-non-unions, chronic pain or tumor of the supraclavicular fossa after management from traditional bonesetters and other hospitals. Some of these patients also felt that their shoulder strength and motion had reduced compare to pre-injury and their contralateral shoulder. Regardless of the fracture history (old or new) all patient were clinically and radiographically evaluated. A total of 876 fracture cases were recorded during the period of the study of which 112 were clavicular fractures. The fractures were classified following the Allman [8] classification based on anatomical site. Treatment was done using the surgical procedure of open reduction and internal fixation (ORIF) based on fracture displacement, mal-union and non-union. Clinical and radiographic assessments of the patients (AN AP Chest X-Ray) were done before surgery, and 1,2, and 3 months after surgery. The study was approved by the ethics committee for human research of the school of medicine and health sciences of the University for Development Studies, Tamale.

\subsection{Statistical Analysis}

The results are expressed as means \pm SD. Chi-square test was used to determine whether there was correlation between the site of fracture as well as the fracture history, age and gender. A level of $\mathrm{P}<0.05$ was considered as statistically significant. Graphpad prism version 5.00 for windows was used for all statistical analysis (Graphpad Software, San Diego California USA, www.graphpad.com).

\section{RESULTS}

The study subjects included 22 females (19.6\%) and 90 males (81.4\%). The mean age of the subjects in years was $34.1 \pm 11.7$ (mean \pm SD), $31.8 \pm 18.6$ for females and $34.5 \pm 10.1$ 
for males. The incidence of clavicular fracture over the study period was 112 out of 876 fracture cases representing $12.8 \%$ of all fracture cases. Majority of the subjects $(71.4 \%)$ with clavicular fractures were between the ages of 21 to 40 years as shown in Table 2. Old cases constituted about $58.9 \%$ whiles only $41.1 \%$ of the cases were fresh fractures that had been brought from road traffic accident site and not been attended to either by the traditional bonesetters or by any hospital. Most of the old cases were likely to be men within the age brackets of 21 to 40 as shown in Table 2. About $70 \%$ of the old cases were people who had received management from traditional bonesetters with the remaining $30 \%$ being management obtain from district hospital/Health centers. The new cases were all fractures that had displacement of at least 2 centimeters with a mean of $2.9 \mathrm{~cm}$ (range, $2.3 \mathrm{~cm}$ to 3.4 $\mathrm{cm})$.

The fracture occurred on the clavicle of the right upper limb in $60.7 \%$ of the patients $(n=68)$, $62.2 \%$ of the males $(n=56 / 90)$ and $59.1 \%(n=13 / 22)$ of the females. In $88(78.6 \%)$ of the subjects the fracture occurred at the level of the middle third and in $24(21.4 \%)$ subjects the fractures occur at the distal third as shown in Table 3. Male subjects were more likely to have a fracture occurring at the middle third $(p=0.02)$ compare to female as shown in Table 2.

The major cause of clavicular fracture in this study was road traffic accident constituting $83.0 \%(93 / 112)$ followed by a fall from height $11.6 \%$ (13/112) and other causes $(5.4 \%)$ which included direct blow to the clavicle, a fall on a level ground and agricultural related activities (Table 1).

Table 1. Cause of clavicular fracture among the subjects

\begin{tabular}{lll}
\hline Cause of injury & No. of cases & $\%$ of patients \\
\hline Road traffic accident & 93 & 83 \\
Fall from a height & 13 & 11.6 \\
Others & 6 & 5.4 \\
Total & 112 & 100.0 \\
\hline
\end{tabular}

Table 2. Fracture history by age and gender

\begin{tabular}{llll}
\hline Age & No. (\%) & Fresh & Old \\
\hline $10-20$ & $10(8.9)$ & 6 & 4 \\
$21-30$ & $41(36.6)$ & 16 & 25 \\
$31-40$ & $39(34.8)$ & 15 & 24 \\
$41-50$ & $14(12.5)$ & 6 & 8 \\
$>50$ & $8(7.2)$ & 3 & 5 \\
Gender & & & \\
Male & $90(80.4)$ & 32 & $58^{*}$ \\
Female & $22(19.6)$ & 14 & 8 \\
Total & $112(100.0)$ & 46 & 66 \\
\hline & & &
\end{tabular}

All the fractures were isolated clavicular fractures without other skeletal involvement. The mean time of union after surgery was 4 weeks (range, 3 to 6 weeks). There were no complications, no infections and mal-union as well as nonunion in all the patients treated operatively. In all no complain of pain at the last follow-up and loss of sensation or 
numbness. One year after treatment about $50 \%$ of the patients came for plate removals for aesthetic concerns and all the removals were an eventful. On the average the patients return to normal activity 8 weeks from the time of the injury regardless of whether the case was an old fracture initially treated conservatively prior to surgery or a new case that was managed surgically.

Table 3. Site of fracture and gender

\begin{tabular}{|c|c|c|c|c|c|c|}
\hline \multirow[t]{2}{*}{ Site of Fracture } & \multicolumn{2}{|c|}{ Female } & \multicolumn{2}{|c|}{ Male } & \multicolumn{2}{|c|}{ Total } \\
\hline & $\mathbf{n}$ & $\%$ & $\mathbf{n}$ & $\%$ & $\mathbf{n}$ & $\%$ \\
\hline Middle Third & 9 & 40.9 & 15 & 16.7 & 24 & 21.4 \\
\hline Distal Third & 13 & 59.1 & $75^{*}$ & 83.3 & 88 & 78.6 \\
\hline Total & 22 & & 90 & & 112 & \\
\hline
\end{tabular}

\section{DISCUSSION}

The general incidence of clavicular fracture in this study was found to be $12.8 \%$ which is relatively higher compare to previous reports by Robinson [4] and Steve et al [5] who have reported clavicular fracture incidence to be around $5-10 \%$. The difference could be attributed in part to the difference in the study population in addition to the high number of road traffic accidents in this part of the world as has been reported [9]. The fracture was more likely to occur in men than female and in men it was more common among under 40 years of age. This finding is in conformity with similar studies which have also found that clavicular fracture are more frequent in younger men than females who usually have clavicular fracture spread out evenly throughout all age groups $[10,11]$. In this part of the world the male gender is dominant in almost every physical activity including driving which may explain the high incidence among men. The most fractured part of the clavicle from this study was the middle third. According to Andermahr et al. [12], the middle third is the thinnest part of the clavicle and is located directly under the skin with little soft tissue or muscle attachment. It is therefore very vulnerable to direct and indirect trauma and this explains the high frequency of fractures in the middle third as reported in the present study which is similar to the findings of other authors [10]. The curvature of a bone, its shape as well as thickness has also been shown to influential parameters to its fracture [13] and this could also be responsible for the high incidence of middle third fractures of the clavicle.

The causes of clavicular fracture range from high impact injuries including road traffic accident fall from a height and a common fall on a level ground [10]. In this study the major cause of the clavicular fracture was road traffic accident followed by a fall from a height. In the Northern part of Ghana the main mode of transportation is motor cycles and bicycles. The few cars compete for space with the motor cycles and bicycles on narrow roads which frequently result in accidents. Earlier studies have shown that every year more than 1.17 million people die in road accidents around the world and about 70 percent of these deaths occur in developing countries including Ghana. According to these studies motor traffic accidence accounted for about $20.0 \%$ fatalities in Ghana. In the Upper West region of Ghana, it is $14.7 \%$ [9]. Reductions of these road traffic accidents may invariable reduce the number of fractures in general and clavicular fractures in particular.

The finding of this study indicates that majority of patients with clavicular fracture will sort for treatment by non-surgical means either from non-orthopaedics surgeons and other clinicians or from traditional bonesetters before consulting the Orthopaedic Surgeon only when the 
desire results are not achieve. Fractures of the clavicle has been in the sphere of nonoperative treatment for a long time but recent studies have shown that the rate of malunion or nonunion after nonoperative treatment might well be much higher than previously shown especially among young adults [10]. In this part of the world clavicular fractures like other fractures are usually managed using the Figure-of-eight or by traditional bonesetters. This practice could be accountable for the high number of mal-union or nonunion reported in the present study as old cases. The old cases were all due to malunion and nonunion leading to unsatisfactory functional results. The question therefore arises as to the relevance of the traditional figure-of-eight for management of clavicular fracture in this part of the world where majority of the fractures are displaced and comminuted which are compounded by mishandling either at the accident site or during transport from the accident site to the health institution due to non availability of the Ambulance system. In addition there have been newer operative techniques with minimal invasion and with better recovery time and functional outcomes compare to the traditional figure-of-eight as has been shown by other studies $[14,15]$. It is the view of the authors that operative management of clavicular fractures in this part of the world may be the way to go since patients who underwent management by both the traditional figure-of-eight and the Traditional bonesetter methods presented with unsatisfactory functional outcomes whereas all the cases that were managed operatively had good functional outcomes without any complications.

\section{CONCLUSION}

The incidence of clavicular fracture is relatively high in the northern part of Ghana and the most affected part of the clavicle is the middle third which occurs mostly among young men under the age of 40 years. The relevance of the traditional bonesetters and the use of the figure-of-eight in management of clavicular fracture among active population such as in the present study need further investigations because of the high incidence of nonunion or malunion associated with it.

\section{CONSENT}

Authors declare that written informed consent was obtained from the patients for publication of this article and accompanying images.

\section{ETHICAL APPROVAL}

All authors hereby declare that the Protocol and procedures have been examined and approved by the Committee for ethics and Human research of the School of Medicine nad Health Science, University for Development Studies and the Tamale Teaching Hospital, Tamale and have therefore been performed in accordance with the ethical standards laid down in the 1964 Declaration of Helsinki.

\section{ACKNOWLEDGEMENTS}

The authors will like to thank all the staff and patients of Tania Specialist Hospital in Tamale, Ghana for their support during the study. 


\section{COMPETING INTERESTS}

Authors have declared that no competing interests exist.

\section{REFERENCES}

1. Moore KL. Clinically Oriented Anatomy. 3 edition. Baltimore: Williams and Wilkins; 1992.

2. Chalidis B, Sachinis N, Samoladas E, Dimitriou C, Christodoulou A, Pournaras J. Acute management of clavicle fractures. A long term functional outcome study. Acta Orthop Belg. 2008;74(3):303-307.

3. Kim W, McKee MD. Management of acute clavicle fractures. Orthop Clin North Am. 2008;39(4):491-505, vii.

4. Robinson CM: Fractures of the clavicle in the adult. Epidemiology and classification. $J$ Bone Joint Surg Br. 1998;80(3):476-484.

5. Steve W, Kotelnicki JJ, Herbert OB, Kevin GM. The Management of clavicular fractures. The Surgical Patient. 2009;19(9):50-56.

6. Edelson G, Saffuri H, Vigder F, Obid E, Nudelman A. Unusual lateral clavicle fracture dislocation: case reports and museum specimens. Clin Orthop Rel Res. 2005;439:274-279.

7. Zlowodzki M, Zelle BA, Cole PA, Jeray K, McKee MD. Evidence-Based Orthopaedic Trauma Working Group. Treatment of acute midshaft clavicle fractures: systematic review of 2144 fractures: on behalf of the Evidence-Based Orthopaedic Trauma Working Group. J Orthop Trauma 2005;19(7):504-507.

8. Allman FLJ. Fractures and ligamentous injuries of the clavicle and its articulation. $\mathrm{J}$ Bone Joint Surg Am. 1967;49(4):774-784.

9. Afukaar FK, Antwi P, Ofosu-Amaah S. Pattern of road traffic injuiries in Ghana: Implication for control. Injuiry Control and Safety Promotion. 2003;10(1-2):69-76.

10. Smekal V, Oberladstaetter J, Struve P, Krappinger D. Shaft fractures of the clavicle: current concepts. Arch Orthop Trauma Surg. 2009;129:807-815.

11. Haider IZ, Khan TA. Clavicle fractures; Incidence a two years study. Professional Med J. 2010;17(2):325-327.

12. Andermahr J, Jubel A, Elsner A, Johann J, Prokop A, Koebke KER. Anatomy of the clavicle and the intramedullary nailing of midclavicular fractures. Clinical Anatomy. 2007;20(1):48-56.

13. Duprey S, Bruyere K, Verriest JP. Influence of geometrical personilization on the simulation of clavicle fractures. Journal of Biomechanics. 2008;41(1):200-207.

14. Judd DB, Pallis MP, Smith E, Bottoni CR. Acute operative stabilization versus nonoperative management of clavicle fractures. Am J Orthop (Belle Mead NJ). 2009;38(7):341-345.

15. Safran $O$, Mosheiff $R$, Mattan $Y$, Liebergall $M$. [Surgical repair of fractures of the clavicle in the adult]. Harefuah. 1997;133(1-2):16-19, 79.

(c) 2013 Callistus and Abass; This is an Open Access article distributed under the terms of the Creative Commons Attribution License (http://creativecommons.org/licenses/by/3.0), which permits unrestricted use, distribution, and reproduction in any medium, provided the original work is properly cited.

Peer-review history:

The peer review history for this paper can be accessed here: http://www.sciencedomain.org/review-history.php?iid=205\&id=12\&aid=1347 